\title{
Transmission and group-delay characterization of coupled resonator optical waveguides apodized through the longitudinal offset technique
}

\author{
J. D. Doménech, * P. Muñoz, and J. Capmany \\ Optical and Quantum Communications Group, ITEAM Research Institute, Universidad Politécnica de Valencia, \\ Camí de Vera s/n, 46020 Valencia, Spain \\ *Corresponding author: jodogo@iteam.upv.es
}

Received September 21, 2010; revised November 22, 2010; accepted December 3, 2010; posted December 8, 2010 (Doc. ID 135526); published January 6, 2011

In this Letter, the amplitude and group delay characteristics of coupled resonator optical waveguides apodized through the longitudinal offset technique are presented. The devices have been fabricated in silicon-on-insulator technology employing deep ultraviolet lithography. The structures analyzed consisted of three racetracks resonators uniform (nonapodized) and apodized with the aforementioned technique, showing a delay of $5 \pm 3$ ps and $4 \pm 0.5 \mathrm{ps}$ over 1.6 and $1.4 \mathrm{~nm}$ bandwidths, respectively. (c) 2011 Optical Society of America

OCIS codes: $130.3120,230.5750$.

Coupled resonator optical waveguides (CROWs) [1] have been extensively studied in the past [2,3]. It is well known that the CROW response presents ripples when the devices are designed for all the couplers to have the same coupling constant. The method to overcome ripples and produce boxlike filtering responses is to set different coupling constants for the couplers, following wellknown windowing techniques used in digital filter design [4]; however, this is the most complicated part from a fabrication perspective. The control over the coupling constants of CROW devices has been done typically by changing the lateral distance between consecutive resonators at a scale of tens of nanometers [5]. To achieve high precision, fabrication techniques as electron-beam patterning are required, at the cost of less device yield per time compared to other techniques, such as photolithography, which in turn have worse resolution (hundreds of nanometers).

In our previous work, we have presented the apodization of CROWs through a novel technique, the longitudinal offset technique [6], where the change in the coupling constants for each cavity in the CROW structure is accomplished by applying a longitudinal offset between the resonators, instead of the conventional transversal offset. This technique alleviates the fabrication requirements, as the change in each stage of the CROW can be 2 orders of magnitude higher than with the conventional techniques. Therefore, it is suitable for mass production fabrication procedures, such as photolitographic systems [7]. In this Letter, we present, to the best of our knowledge, the first practical demonstration of the aforementioned longitudinal offset technique.

Figure 1 shows scanning electron microscope (SEM) images of the uniform and apodized CROW devices, respectively, fabricated in silicon-on-insulator technology using deep ultraviolet (DUV) lithography [8]. The silicon photonic wire waveguide, on top of a $2-\mu$ m-thick BOX layer, is $530 \mathrm{~nm}$ wide by $220 \mathrm{~nm}$ thick in order to ensure TE monomode propagation. The racetracks have a bend radius $R=5 \mu \mathrm{m}$ and a straight section $L_{s}=53.3 \mu \mathrm{m}$ in both devices. The measured linear propagation loss in such photonic wires is $6 \pm 1 \mathrm{~dB} / \mathrm{cm}$, and the group index around the $1.55 \mu \mathrm{m}$ wavelength is $n_{g} \simeq 4.25$ and has been derived from the free spectral range (FSR) of the CROW devices. The measured FSR near the $1.55 \mu \mathrm{m}$ wavelength was $4.09 \mathrm{~nm}$. The gaps between cavities in the uniform and apodized CROWs have been set to $150 \mathrm{~nm}$, a safe value for the $193 \mathrm{~nm}$ DUV lithography employed. In the case of the uniform CROW, all the racetracks have the same coupling length and, therefore, the same coupling constant $\left(K_{1}=K_{2}=K_{3}=K_{4}=0.48\right)$, while the couplings in the apodized CROW have been set symmetrically: the longitudinal offset applied to the first and last racetrack is the same, and the resulting coupling constants have a value of $K_{1}=K_{4}=0.86$. A different offset has been applied to the central racetrack and, as a result, $K_{2}=K_{3}=0.54$, as proposed in our previous theoretical work $[\underline{4}, 6]$. Translating the coupling values to physical parameters, for a straight coupling section of $53.3 \mu \mathrm{m}$, the actual coupling length is even longer and is given by [9]

$$
L_{c}=L_{s}+2 \cdot R \cdot \arccos \left(1-\frac{d_{c}-d}{2 \cdot R+w}\right),
$$

where $L_{s}$ is the straight coupling section, $R$ is the bend radius, $d$ is the gap between resonators, $w$ is the waveguide width, and $d_{c}$ is the distance between waveguides where light starts to couple in the bent section, which, for this waveguide cross section and gap, is estimated to be $0.4 \mu \mathrm{m}$. Figure 2 illustrates the longitudinal offset and the conventional apodization techniques, showing the coupling constant between resonators versus the coupling length for several gap distances, $d$, between resonators. The black square represents the nominal coupling value for the uniform CROW device given a fixed coupler length and $d$. The CROW apodization requires increasing $K$ progressively; therefore, the choice of a coupler length of $53.3 \mu \mathrm{m}$ ensures that we are in the negative slope of the coupling, and applying a longitudinal offset implies an increase in the coupling constant. Apodization requires $K_{1}=K_{4}=0.86$ and $K_{2}=K_{3}=0.54$ as outlined later. This translates, as shown in Fig. 2 , to a longitudinal offset of $10.56 \mu \mathrm{m}\left(L_{c}-L_{c 2}\right)$ and $2.42 \mu \mathrm{m}\left(L_{c}-L_{c 1}\right)$, 


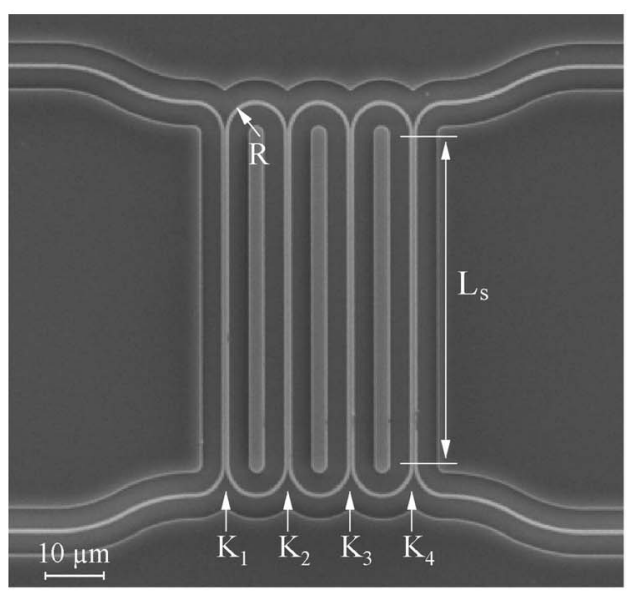

(a)

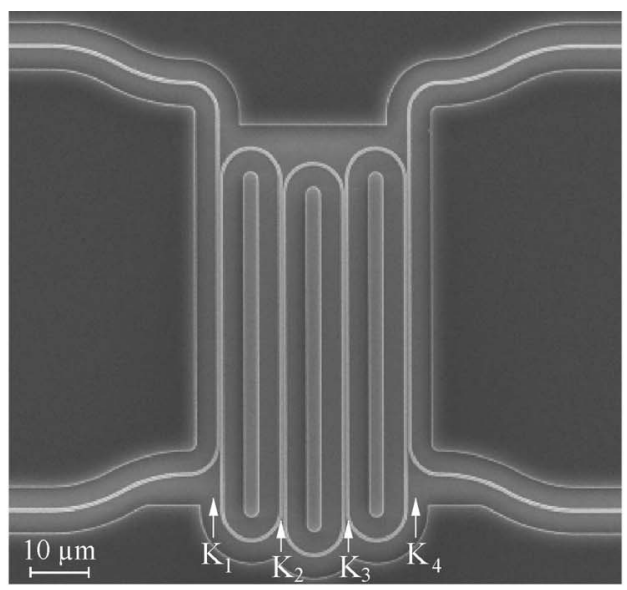

(b)

Fig. 1. SEM images of (a) a three-racetracks CROW uniform and (b) apodized with the longitudinal offset technique. $R$ is the bend radius of the racetracks, $L_{s}$ is the straight section length, and $K_{i}$ are the coupling constants for each section.

respectively. Conversely, the same apodization through a change of the coupler gap would require gaps of $153 \mathrm{~nm}$ $\left(d_{1}\right)$ and $170 \mathrm{~nm}\left(d_{2}\right)$ for fixed $L_{c}$. Comparing the required changes with both techniques, it is clear that the longitudinal offset technique requires less precision and is less sensitive when setting the coupling constants.

The power and group delay transfer function were measured for the devices in Fig. 1, employing the setup shown in Fig. 3 [10]. The results are given in Fig. $\underline{4}$. A tunable laser (ANDO AQ4321D, $\lambda=1520-1620 \mathrm{~nm}$ ) was externally modulated with a $1 \mathrm{GHz}$ signal. At the output of the modulator, the light was amplified with an erbium-doped fiber amplifier. The polarization was controlled both at the input of the modulator and at the input of the chip. The signal must be realigned in polarization, because grating couplers [11] are used for in/ out coupling light into the device under test and have been optimized for TE propagation. At the output of the chip, the signal was then photodetected. Central in this setup is the microwave networks analyzer (Agilent, E8364A), which drives the modulator and receives the signals from the photodetector. It measures the ampli-

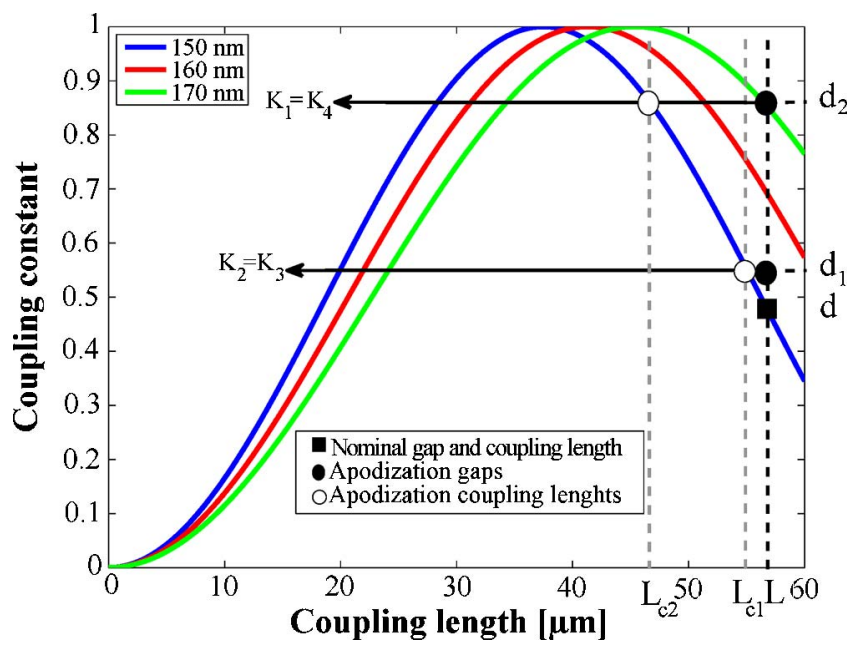

Fig. 2. (Color online) Coupling calculation between resonators, illustrating the longitudinal offset and the conventional apodization techniques. tude and phase of the signal. The group delay is expressed in terms of the phase as follows:

$$
\tau_{g}(\lambda)=\frac{\phi_{d}(\lambda)+\phi_{0}}{\omega_{m}}
$$

where $\phi_{d}(\lambda)$ is the measured phase of the signal, $\phi_{0}$ is a constant phase term introduced by the setup without the resonant structures, and $\omega_{m}$ is the modulation angular frequency. The term $\phi_{0}$ has been obtained by averaging several measures done on test straight waveguides employing the same setup.

Figure 4 shows the measured amplitude and group delay (gray/red curves) of the devices compared with the theoretical responses (black curves) calculated with the transfer matrix method described elsewhere [12-14], where losses and the coupler length were taken into account. In the case of the uniform CROW [Fig. 4(a)], the measured transmission passband exhibits big ripples that have natural correspondence with the group delay. These ripples are due to the fact that all the coupling constants are equal [4]. The measured transmission presents ripples of $3.5 \overline{\mathrm{dB}}$. A mean delay of $5 \mathrm{ps}$ has been measured with ripples of $\pm 3 \mathrm{ps}$ in a $3 \mathrm{~dB}$ bandwidth of $1.6 \mathrm{~nm}$, as predicted by the transfer matrix calculations. For the offset apodized CROW [Fig. 4(b)], a flat response in transmission with ripples of less than $1 \mathrm{~dB}$, due to the apodization of the structure, has been measured, and a delay in the passband of about $4 \mathrm{ps}$ with reduced ripples of $\pm 0.5 \mathrm{ps}$ in a $3 \mathrm{~dB}$ bandwidth of $1.4 \mathrm{~nm}$.

To summarize, we demonstrated how the apodization of CROWs through the longitudinal offset technique is as effective as through conventional techniques, reducing the accuracy required for fabrication. The measured

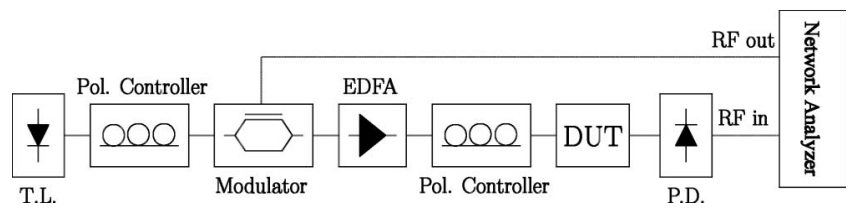

Fig. 3. Group-delay characterization setup. Continuous lines, optical signals; dashed lines, electrical signals. 


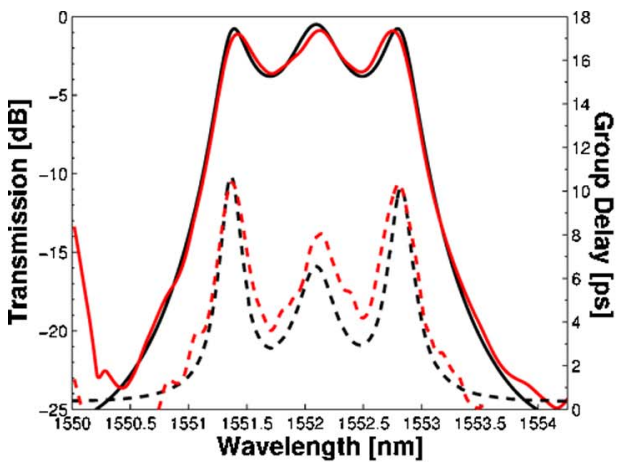

(a)

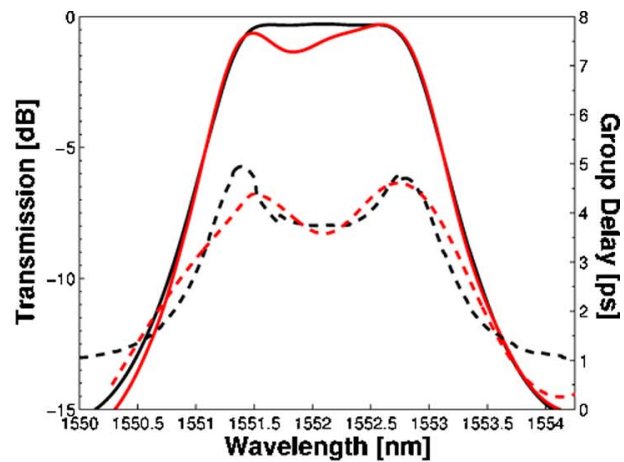

(b)

Fig. 4. (Color online) Power transmission and group-delay responses of (a) a three-racetracks CROW device uniform and (b) offset apodized CROW device. Black curves (continuous for the amplitude and dashed for the group delay) represent the theoretical responses, and gray/red curves represent the measured responses.

responses are in good agreement with the theoretical predictions extracted with the transfer matrix method. As expected, the ripples within the passband transmission and group delay are reduced through apodization.

This work has been funded through the Spanish Plan Nacional de I+D+i 2008-2011 project TEC200806145/TEC Coupled Resonator Optical Waveguides eNgineering (CROWN). J. D. Doménech acknowledges the Beca de Formacion de Personal Investigador (FPI) research grant BES-2009-018381.

\section{References}

1. A. Yariv, Y. Xu, R. K. Lee, and A. Scherer, Opt. Lett. 24, 711 (1999).

2. J. K. Poon, J. Scheuer, Y. Xu, and A. Yariv, J. Opt. Soc. Am. B 21, 1665 (2004).

3. J. K. Poon, L. Zhu, G. A. DeRose, and A. Yariv, Opt. Lett. 31, 456 (2006).

4. J. Capmany, P. Munoz, J. D. Domenech, and M. A. Muriel, Opt. Express 15, 10196 (2007).
5. F. Xia, M. Rooks, L. Sekaric, and Y. Vlasov, Opt. Express 15, 11934 (2007).

6. J. D. Domenech, P. Munoz, and J. Capmany, Opt. Express 17, 21050 (2009).

7. W. Bogaerts, R. Baets, P. Dumon, V. Wiaux, S. Beckx, D. Taillaert, B. Luyssaert, J. V. Campenhout, P. Bienstman, and D. V. Thourhout, J. Lightwave Technol. 23, 401 (2005).

8. URL: www.epixfab.eu.

9. F. Xia, L. Sekaric, and Y. A. Vlasov, Opt. Express 14, 3872 (2006).

10. C. K. Madsen and J. H. Zhao, Optical Filter Design and Analysis: a Signal Processing Approach (Wiley, 1999).

11. D. Taillaert, F. Van Laere, M. Ayre, W. Bogaerts, D. Van Thourhout, P. Bienstman, and R. Baets, Jpn. J. Appl. Phys. 45, 6071 (2006).

12. J. Capmany and M. A. Muriel, J. Lightwave Technol. 8, 1904 (1990).

13. J. Poon, J. Scheuer, S. Mookherjea, G. Paloczi, Y. Huang, and A. Yariv, Opt. Express 12, 90 (2004).

14. Y. M. Landobasa, S. Darmawan, and M. K. Chin, IEEE J. Quantum Electron. 41, 1410 (2005). 